\title{
Agent-based Model for Environmental Awareness and Extended Producer Responsibility in Developing Countries
}

\author{
Modelo basado en agentes para simular la conciencia ambiental y responsabilidad \\ ampliada del productor en países en vías de desarrollo
}

\author{
J. D. Galarcio-Noguera iD ; P. A. Maya-Duque iD , G.L. Ramírez-Córdoba iD; Y.F. Ceballos iD
}

\begin{abstract}
To make an effective transition towards a circular economy, we must have the responsibility and active participation of all the stakeholders involved, including customers. In this work, we seek to develop an agent-based model to describe and analyze an environmental awareness system in a population, whose behavior is based on an indicator that can change by agent's interaction and modify its consumption habits. The model is described using the ODD $+D$ protocol, and we use Netlogo software for coding. After model validation process, its results are analyzed to understand how can environmental awareness level, consumption habits and social skills of each individual influence on the average environmental awareness level of a heterogeneous population, which makes simple the identification of emerging behaviors and the forecasting of the potentially recyclable waste according to the quality in the separation at source.
\end{abstract}

Index Terms - Agent Based Modeling (ABM), environmental management, extended producer responsibility (EPR), social factors, sustainable development.

Resumen- Para hacer una transición efectiva hacia una economía circular, se debe tener la responsabilidad y la participación de todas las partes interesadas involucradas, incluidos los clientes. En este trabajo, se busca desarrollar un modelo de simulación basado en agentes para describir y analizar un sistema de conciencia ambiental en una población, cuyo comportamiento se basa en un indicador que puede cambiar por la interacción del agente y modificar sus hábitos de consumo. El modelo se describe utilizando el protocolo ODD + D, y se utiliza el software Netlogo para la codificación. Después del proceso de validación del modelo, sus resultados se analizan para entender cómo puede el nivel de conciencia ambiental, los hábitos de consumo y las habilidades sociales de cada individuo influir en el nivel medio de conciencia ambiental de una población heterogénea, lo que simplifica la identificación de comportamientos emergentes y la previsión de los residuos potencialmente reciclables de acuerdo con la calidad en la separación en origen.

This manuscript was submitted on May 15, 2020 and accepted for publication on September 06, 2020.

J. D. Galarcio-Noguera is an Industrial Engineer and a student in master's engineering program of Universidad de Antioquia Colombia (e-mail: jdavid.galarcio@gmail.com).

P. A. Maya is a Ph.D. in operations research of University of Antwerpen and Full-time professor at the industrial department, engineering faculty of Universidad de Antioquia Colombia (e-mail: pablo.maya@udea.edu.co).
Palabras claves - Modelado basado en agentes (ABM), desarrollo sostenible, factores sociales, gestión ambiental, responsabilidad extendida del productor (EPR).

\section{INTRODUCTION}

SINCE 1971, the impact of population growth on the Senvironment has been a topic of interest [1]. This growth has been seen as a degrading factor of environmental quality: as the pressure on the assimilative capacity of the environment increases, air and water pollution, along with the amount of solid waste generated also increase [2]. In general, waste generation is expected to increase from 2.0 billion tons per year reported in 2016 to 3.4 billion in 2050, given the estimated population growth [3]. Consequently, it is important to recognize that society is in a waste crisis, demanding the development and implementation of tools and strategies to improve the current situation [4].

The circular economy (CE) is considered as a potential solution, oriented to protect the environment without becoming an obstacle to economic growth [5], [6]. Accordingly, the United Nations (UN) has included responsible production and consumption among the sustainable development objectives [7], promoting the transition towards CE [6]. To do so, a concept is known as extended producer responsibility (EPR) [8] has emerged, calling for companies to take responsibility for the environmental impacts generated by their products throughout their entire life cycle.

Packages are part of the waste that people generate every day. In this sense, the amount of packaging in the waste stream and the difficulty to manage it properly have promoted the development of related policies in different countries [9]. In Europe, an action plan related to reuse, recycling and disposal was developed, including concepts like extended responsibility and eco-design of products [10]. Colombia has considered responsible packaging management as one of its extended

G.L. Ramírez-Córdoba is a Ph.D. in Contabilidad y Finanzas of Universidad de Zaragoza and Full-time professor at the industrial department, engineering faculty of Universidad de Antioquia Colombia (e-mail: glorial.ramirez @udea.edu.co).

Y.F. Ceballos is a Ph.D. in engineering of Universidad Nacional de Colombia and Full-time professor at the industrial department, engineering faculty of Universidad de Antioquia Colombia (e-mail: yony.ceballos@udea.edu.co). 
responsibility schemes, and has designed standards for the environmental management of paper, cardboard, plastic, glass and metal packaging [11], [12].

One of the aspects associated with the implementation of the REP scheme for packaging in Colombia is separation at source by consumers, an effective way to increase recycling volumes that has proved to be successful in developed countries [13]. This not only contributes to increasing the volume of potentially recyclable material, but also creates a favorable scenario for companies to meet the goals imposed by the EPR regulations [12]. It should be noted that although separation at source is successful in developed countries, it requires the existence of a civic culture of environmental awareness that is not necessarily present in developing countries [14].

To optimize the process of separation at source, it is essential to have the commitment and active participation of all the actors involved, including consumers. Also, understanding how the environmental awareness level, consumption habits and social skills of individuals influence the average environmental awareness level of a heterogeneous population can facilitate the identification of emerging behaviors and forecasting the volume of potentially recyclable waste based on the quality in the separation process. To describe and analyze these types of situations involving heterogeneous individuals making decisions and interacting with each other and their environment, agent-based simulation (ABS) is useful [15].

To design and describe models developed under this simulation paradigm, a protocol that allows for the consideration of general, design concepts and a detailed explanation that guarantees their reproducibility is used. In this case, we will use the ODD $+\mathrm{D}$ protocol, which includes the conventional protocol with an extension that allows describing in a structured way the human decisions, allowing detailing the learning and the adaptability of the agents to their environment [16]. Among the computational tools for SBA problem modeling we find software such as MATSim [17], The Repast Suite [18], Swarm [19] and NetLogo [20].

This paper focuses on the development of an agent-based simulation model to describe and analyze how environmental awareness evolves in a population, considering factors such as the environmental awareness level here on denoted as eco-level. The agents in the model are classified into three groups according to their eco-level. The model considers the proportion of people with high, medium, and low eco-level, a learning rate, the amount of waste generated by each individual and the percentage of potentially recyclable waste according to their eco-level. Also, aspects such as the degree of sociability, power of persuasion and resistance to change are included. In general, the population behaves according to an eco-level indicator that can change depending on the interaction between agents (individuals) and modify their consumption habits and waste separation behaviors at the source. The model represents the weight of recyclable and non-recyclable waste in the simulation time. Once the system has been analyzed, the model is described clearly and precisely to guarantee its replicability through the ODD + D protocol. Besides, the NetLogo software is used to model the system, where an intuitive and useful interface is presented to represent the described problem. This article is divided into 4 sections. The following section describes the model according to the ODD $+\mathrm{D}$ protocol. In section 3 , the steps performed to validate the model and the analysis of the results are shown. Finally, conclusions and future research directions derived from this work are presented in section 4.

\section{MODEL DEVELOPMENT}

The model described below use the ODD + D protocol [16]. For research purpose, the potentially recyclable material frames containers and packaging of paper, cardboard, plastic, glass and/or metal that can be recycled, reused, recovered and/or reintegrated into the value chain. The proportion of this material in all waste generated per person in a unit of time is presented as a percentage, and it is assumed that the rest of the waste does not meet those characteristics.

\section{A. Overview}

\section{1) Purpose}

With this model, we want to understand how environmental awareness level, consumption habits, and individual social skills influence the average eco-level and waste separation behaviors at the source of a population. The latter is measured in terms of the amount of waste properly separated.

This model aims at supporting decision makers and authorities in charge of design policies of extended producer responsibility in different packaging classes, so that, according to the specific characteristics of a population, they could generate and project estimates on the average environmental awareness level and the amount of recycled material. This would allow them to evaluate the relevance and viability of the percentage thresholds that they raise in terms of recovered material and the deadlines that are foreseen for in planning of governmental policies.

\section{2) Entities, state variables and scales}

The model consists of a single human agent that represents an individual in the population. Each human has the following characteristics: environmental awareness level (eco-level), amount of waste generated, sociability radius, persuasiveness, and level of resistance to change. All variables are endogenous since none are affected by external alterations. Global variables are included such as the amount of recoverable waste separated in a correct way (RWSC), the amount of recoverable waste separated in an incorrect way (RWSI) and the amount of nonrecoverable waste $(\mathrm{NW})$ in kilograms. In addition, there are variables such as the number of people within the range of low (NPLe), medium (NPMe) or high (NPHe) eco-level. Also, each group have a percentage of potentially recyclable waste according to its consumption habits. Finally, we have a learning rate to improve the eco-level at individuals, and the average environmental awareness level of the population (average ecolevel). Table 1 shows the range of each variable.

For its part, space implicitly represents a closed place where humans frequent and interact. It could represent, for example, the campus of a university or a delimited geographical area (Figure 1(a)). Space allows one or another type of 
neighborhood at the same time: according to Moore or von Neumann neighbors (Figure 1(b) and (c)).

TABLE I

GENERAL VARIABLE INFORMATION

\begin{tabular}{|c|c|c|}
\hline Type & Variable & Range \\
\hline Agent & eco-level & {$[0,10] \in \mathrm{R}$} \\
\hline Agent & Waste generated $(\mathrm{Kg})$ & $(0,+\infty) \in \mathrm{R}$ \\
\hline Agent & sociability radius & {$[1,10] \in Z$} \\
\hline Agent & Persuasiveness & $(0,1] \in \mathrm{R}$ \\
\hline Agent & resistance to change & $(0,1] \in \mathrm{R}$ \\
\hline Agent & Color & $\begin{array}{l}\text { Red, Yellow and } \\
\text { Green }\end{array}$ \\
\hline Global & $\begin{array}{l}\text { Amount of recoverable waste } \\
\text { separated in a correct way (RWSC) }\end{array}$ & $(0,+\infty) \in \mathrm{R}$ \\
\hline Global & $\begin{array}{l}\text { Amount of recoverable waste } \\
\text { separated in an incorrect way (RWSI) }\end{array}$ & $(0,+\infty) \in \mathrm{R}$ \\
\hline Global & $\begin{array}{l}\text { Amount of non-recoverable waste } \\
\text { (NW) }\end{array}$ & $(0,+\infty) \in \mathrm{R}$ \\
\hline Global & $\begin{array}{l}\text { Number of people with low eco-level } \\
\text { (NPLe) }\end{array}$ & {$[1,100] \in \mathrm{Z}$} \\
\hline Global & $\begin{array}{l}\text { Percentage of potentially recyclable } \\
\text { waste for NPLe (rwNPLe) }\end{array}$ & {$[0,1] \in \mathrm{R}$} \\
\hline Global & $\begin{array}{l}\text { Number of people with low eco-level } \\
\text { (NPMe) }\end{array}$ & {$[1,100] \in \mathrm{Z}$} \\
\hline Global & $\begin{array}{l}\text { Percentage of potentially recyclable } \\
\text { waste for NPMe (rwNPMe) }\end{array}$ & {$[0,1] \in \mathrm{R}$} \\
\hline Global & $\begin{array}{l}\text { Number of people with low eco-level } \\
\text { (NPHe) }\end{array}$ & {$[1,100] \in Z$} \\
\hline Global & $\begin{array}{l}\text { Percentage of potentially recyclable } \\
\text { waste for NPHe (rwNPHe) }\end{array}$ & {$[0,1] \in \mathrm{R}$} \\
\hline Global & learning rate & {$[0,1] \in \mathrm{R}$} \\
\hline Global & average eco-level & {$[0,10] \in \mathrm{R}$} \\
\hline Global & maximum displacement & {$[1,20] \in \mathrm{Z}$} \\
\hline Space & type of neighborhood & $\begin{array}{l}\text { "Von Neumann" or } \\
\text { "Moore" }\end{array}$ \\
\hline
\end{tabular}

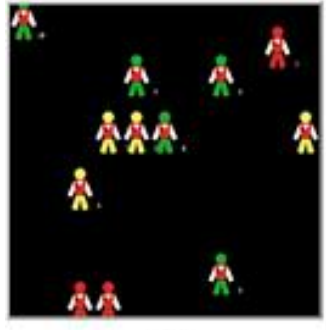

(a)

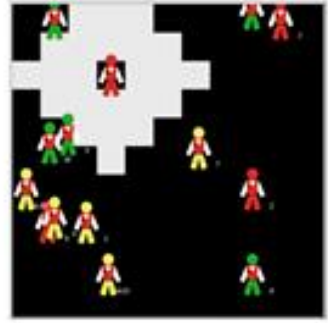

(b)

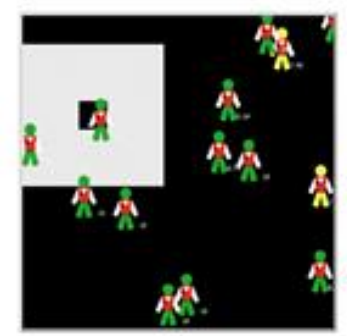

(c)

Fig. 1. (a) Space in which simulated events occur, (b) Von Neumann neighbors with radius $=3$, and (c) Moore's neighbors with radius $=2$.

The sociability range represents how sociable the individual is and gives him the ability to interact with more or fewer neighbors. Finally, each tick represents a unit of time in which humans interact between them within their radius of sociability and perform a displacement. For the purposes of this model, a unit of time represents a day.

\section{3) Process overview and scheduling}

After the model initialization, each human check its neighborhood within its sociability radius according to the type of neighborhood defined for the simulation. If the agent meets another human within its neighborhood, it will act according to its his characteristics.

That is, if the neighbor's eco-level is greater than the eco-level of the agent is, it proceeds to check the next neighbor. If the neighbor's eco-level is lower than the agent eco-level and the agent is part of the low group (eco-level $<3$ ), the agent will do nothing. If the neighbor's eco-level is less than the agent ecolevel and the agent is part of the middle group $(3 \leq$ eco-level $<8$ ), a binary random number is generated, if the number is equal to 1, a persuasion process is initiated that consists in comparing the persuasiveness of the human with the level of resistance to change of its neighbor, if the human persuasiveness is greater than the level of the neighbor, the ecolevel of the neighbor will be increased according to the learning rate that has been set for simulation, otherwise, no action is taken. Finally, if the human is part of the high-level eco-group $(8 \leq$ eco-level $\leq 10)$, it will always start a persuasion process and try to increase the eco-level of its neighbors and its success will depend on the human persuasiveness.

Once the agent human interacts with all its neighbors, an integer random number is generated between 1 and the maximum displacement value defined and the agent moves in any direction according to the value generated. If a human change from one group to another (NPLe, NPMe and NPHe), its consumption habits change. Ideally, it will increase its consumption of products with potentially recoverable waste and will decrease proportionally the consumption of products that are not environment friendly. In the model, this is represented as a modification in the percentage of potentially recoverable waste that the human contributes to the total waste generated in the system.

After performing this procedure for each agent, the following criteria is considered: If the human is part of the NPLe, it is assumed that it does not separate. If the human is part of the $N P M e$, it may or may not separate correctly. Finally, if the agent is part of the $\mathrm{NPHe}$, it will always separate correctly. From the above, the following global variables are updated: $R W S C, R W S I$, $N W$, and average eco-level for the population. The above procedure is shown in Figure 2.

\section{B. Design concepts}

\section{1) Theoretical and empirical background}

The model arises in response to develop tools to test the policies design for extended liability schemes [6], [11], [12], [21], taking into account that environmental awareness cannot be taken for granted in under development countries [14], and that it is possible to analyze the behaviors of heterogeneous and autonomous populations through ABS [16], [22], [23]. In addition, successful models of ABS have been developed that involve environmental awareness and social behaviors [24].

\section{2) Learning}

Humans are increasing their eco-level, so it is possible to affirm that there is learning, which is incorporated into the decision process through the level of resistance to change (an individual can refuse to increase their eco-level). It is not 


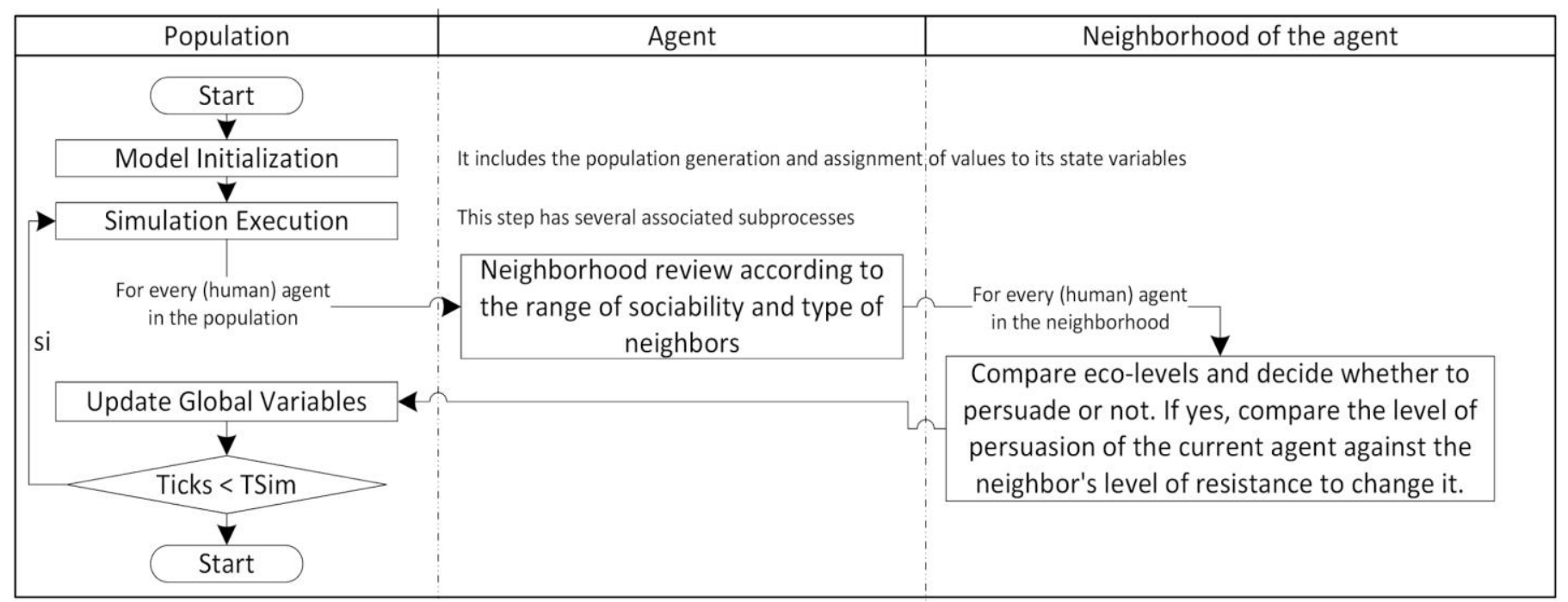

Fig. 2. Simplified process description.

implemented, but collective learning is measured.

3) Individual sensing

In general, humans perceive their eco-level, their belonging to one group or another to make decisions, and neighbors within their sociability radius. When an agent tries to persuade another, it perceives the eco-level and the resistance to change, while the other agent perceives his persuasiveness. The spatial detection scale is local. In addition, the mechanisms for obtaining information are implicitly modelled if the agents know the values of these variables. The costs of cognition or information gathering are not explicitly included.

\section{4) Individual prediction}

In this model, each human must know how many neighbors are within their sociability radius and predict the eco-level of each of their neighbors to determine if they are susceptible to being influenced. According to the group where it belongs, the human may adopt different positions: not persuade - not separate waste, persuade and separate occasionally or always persuade and separate. It could be said that, knowing the change resistance level of a neighbor, they can predict a failed attempt at persuasion, and knowing the eco-level of their neighbor, they can predict an unnecessary or potentially successful persuasion. 5) Interaction

The interactions between humans would occur directly, depending on the sociability radius, the type of neighborhood and the position of each agent in space. The communication between the agents consists of a process of persuasion resistance to change, which refers to the power of conviction that a human has and that it uses to try to persuade, confronted with the resistance that another human imposes to change its way of thinking in terms of environmental awareness, that is, its eco-level and, gradually, its consumption habits. This communication, as already mentioned, is represented by comparing the variable persuasiveness with the level of resistance to change.

6) Collectives

There are three groups that can affect and be affected by the agents. Agents with a low environmental awareness are represented with the color red, have an eco-level between 0 and
3 (not including 3) and are characterized by having little ecological consumption habits, in which the percentage of potentially recoverable waste is necessarily low, and this small percentage is not separated, so it becomes part of the recoverable material not separated or incorrectly separated. In consequence, these humans do not try to persuade others to increase their environmental awareness. Agents with a medium environmental awareness are shown in yellow, their eco-level is between 3 and 8 (not including 8), they have moderately ecological consumption habits then their percentage of potentially recoverable waste is strictly higher than the agents of the first group. The behavior of those agents is variable: in some cases, they are willing to persuade others to increase their ecological awareness, however, they may also decide to assume a passive stance and do not take any action. With respect to classifying the recoverable material they follow the same pattern of behavior, so they contribute both to the recyclable material not separated or incorrectly separated, as well as to the recyclable material properly separated. This way of proceeding is represented by generating a value that can be 0 or 1 , with 1 being the value that indicates a decision in favor of the environment. Finally, we have agents that have a high environmental awareness, are represented with the color green, their eco-level is between 8 and 10, and have highly ecological consumption habits, so the percentage of potentially recoverable waste derived from the products consumed is necessarily high. Them always performs the separation process in an appropriate way, so it contributes to the recyclable material properly separated.

It is important to mention that there is a part of the waste that is not recoverable, therefore, all humans contribute to the recyclable material in a percentage of the total of their generated waste, which would be 1 minus the percentage of potentially recoverable waste applied for each collective. The number of individuals belonging to each group is defined by the researcher on a preliminary basis and changes based on the interactions between the agents.

7) Heterogeneity

As already mentioned, the set of human agents is 
heterogeneous, both in the value of its state variables, and in decision making. This difference is reflected in the decisions that each agent can make according to their degree of environmental awareness.

8) Stochasticity

The model includes random aspects, both in the decision making of the agents (binary random) and in the initialization of the state variables and the model in general. The initialization process will be detailed later.

\section{9) Observation}

The data collected from the simulation includes $R W S C$, $R W S I$, and $N W$ generated by the population. This information is extracted after a tick, which represents a day in our model. Figure 3 (a) shows an example of the model outputs for a 10day simulation. Another important fact is the number of people that make up each group, which allows us to identify how the proportions change as their eco-level changes, and how it is reflected in waste separation behaviors at the source. The way in which an observer perceives that information is presented in Figure 3 (b).
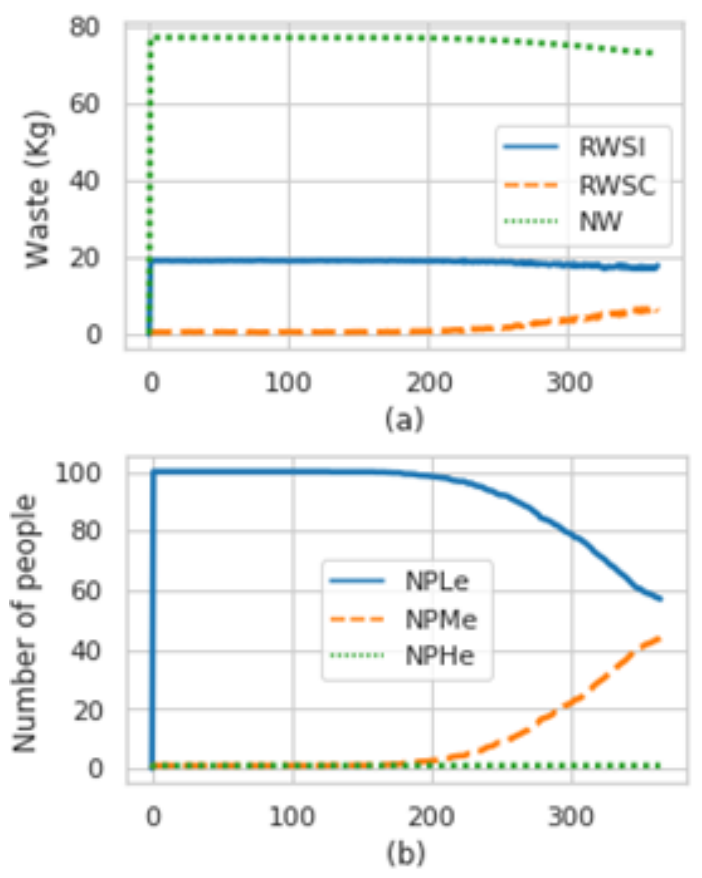

Fig. 3. (a) Discriminated amount of waste from the population $(\mathrm{Kg})$, and (b) Evolution of the number of people per group.

Finally, we have the average eco-level, which is calculated as the average of the eco-levels of all humans. This data is key to our analysis, as it allows us to see how environmental awareness evolves. The key results and the characteristics of the model outputs are emerging, taking into account that, although the average ecological level is expected to grow gradually, it is difficult to know in advance in which moment of time it will take for the population to reach to have a mature ecological behavior, how the population composition changes according to the three defined groups and what approximate amount of recyclable waste properly separated could be expected in an instant of time according to the environmental awareness of the population.

\section{Details}

The model was implemented in the NetLogo software, and the code can be accessed through the authors of this work. With respect to the initialization, its initial state will depend on the population type, allowing to generate the value of the waste generated by each human in ( $\mathrm{kg} / \mathrm{hab}$. / Day) from a normal distribution with a given mean and deviation. For this value, we have used a mean of 0.95 and a deviation of 0.1 . The average value has been obtained from the literature for 2012 [3]; however, the deviation has been empirically fixed.

Also, it is possible to set the number of individuals to be generated and the percentage of potentially recyclable waste (consumption habits) for each group. The number of individuals could be defined according to a proportion of a real population, however, all these values have been empirically defined, respecting the assumption that, at the beginning, NPLe $>\mathrm{NPMe}$ $>$ NPHe for quantity, and rwNPLe < rwNPMe < rwNPHe for the percentage of potentially recyclable waste that would be determined by consumption habits.

In addition, the maximum displacement allowed for all humans can be defined per unit of time, which is generated from a random integer between 1 and this maximum value for everyone in each tick. This value can be modified during simulation and has been empirically set. Another key value that can be established at the beginning and modified during the simulation is the learning rate of individuals. This rate helps us adjust the model to consistent results from the point of view of learning - time.

Finally, we have the values related to the social aspect. The sociability radius, which could be characteristic of everyone from beginning to end or be dynamic in the simulation, in this case, has been set as a characteristic of everyone. For its part, the Moore / Von Neumann type of neighborhood can also be static or modified during simulation. To initialize and execute the model, there are interface controls for the following variables: waste generated (mean and standard deviation), sociability radius, NPLe, rwNPLe, NPMe, rwNPMe, NPHe, rwNPHe, learning rate, maximum offset and type of neighborhood.

With respect to the sub models, these have been throughout this work. Considering that the model and all sub models were designed in the absence of research that associated the factors present in this work, there are no reference values for most of the model parameters, however, the ranges defined for the state variables are found available in Table 1.

\section{RESUlts AND ANALYSIS}

The validation process is performed for a baseline scenario (BS). For this, it is expected that with a large number of people with low environmental awareness and only one person from each of the other groups, the evolution in a simulation year (365 days) will be almost zero for all the model outputs. The parameters are showed in Table 2. According to the results obtained from the simulation, we can say that these fit with the proposed hypothesis for the baseline scenario. 


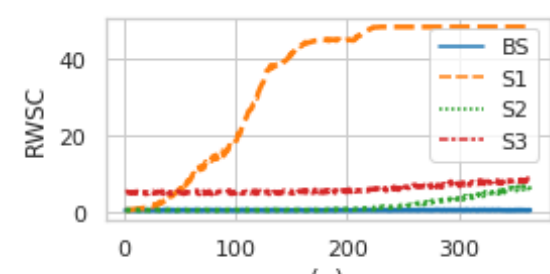

(a)

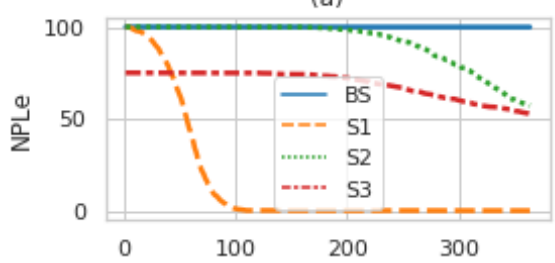

(d)

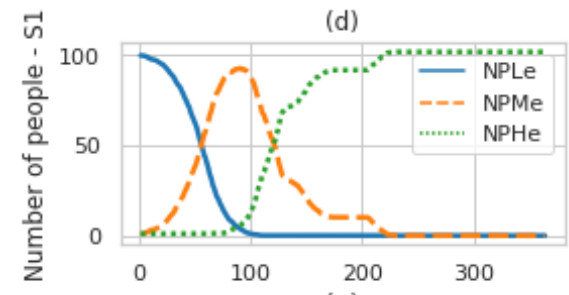

(g)

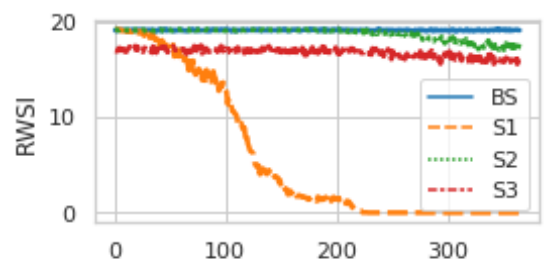

(b)

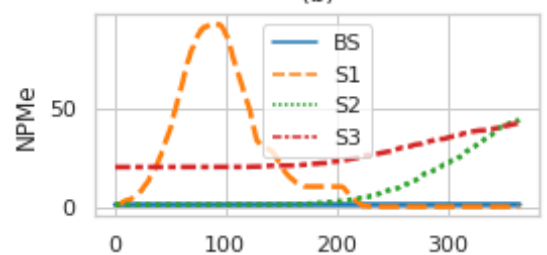

(e)

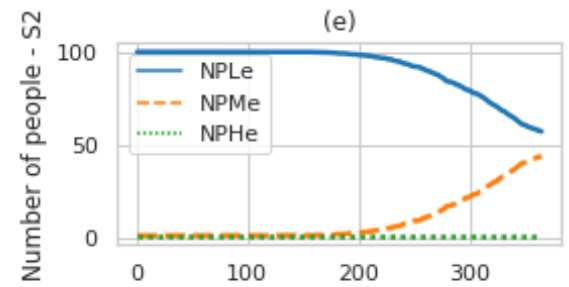

(h)

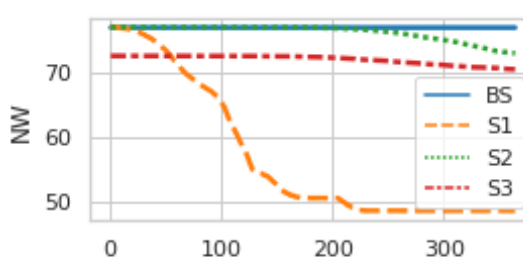

(c)
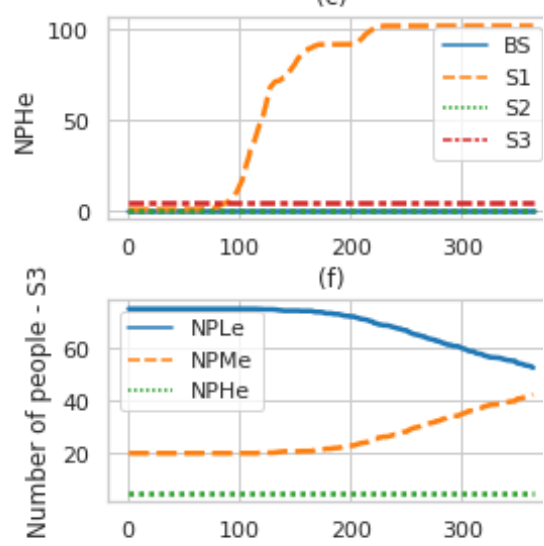

(i)

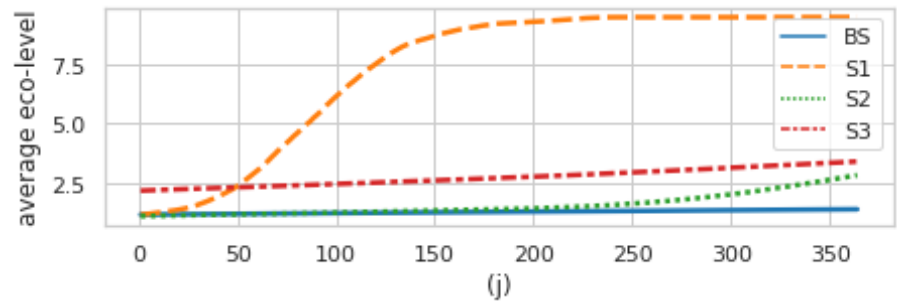

Fig. 4. Results summary by scenario.

As pointed out before, three scenarios to test the model were generated by changing parameters in the baseline scenario. That is shown in the next subsections.

TABLE II

PARAMETERS

\begin{tabular}{lllll}
\hline \multicolumn{1}{c}{ Parameters } & BS & S1 & S2 & S3 \\
& & & & \\
\hline NPLe & 100 & 100 & 100 & 75 \\
NPMe & 1 & 1 & 1 & 20 \\
NPHe & 1 & 1 & 1 & 5 \\
rwNPLe & $20 \%$ & $20 \%$ & $20 \%$ & $20 \%$ \\
rwNPMe & $30 \%$ & $30 \%$ & $30 \%$ & $30 \%$ \\
rwNPHe & $50 \%$ & $50 \%$ & $50 \%$ & $50 \%$ \\
Maximum offset & 7 & 7 & 7 & 4 \\
Learning rate & $3 \%$ & $50 \%$ & $3 \%$ & $3 \%$ \\
Type of neighborhood & $\mathrm{VN}$ & $\mathrm{VN}$ & $\mathrm{VN}$ & $\mathrm{VN}$ \\
Sociability radius & 4 & 4 & 10 & 3 \\
Mean of waste generated by & 0.95 & 0.95 & 0.95 & 0.95 \\
$\begin{array}{l}\text { an agent (Kg/hab./day) } \\
\text { Standard Deviation of waste } \\
\text { generated by an agent }\end{array}$ & 0.1 & 0.1 & 0.1 & 0.3 \\
(Kg/hab./day) & & & & \\
\hline \hline VN: Von Neumann & & & & \\
\hline
\end{tabular}

VN: Von Neumann

Scenario 1 (S1): Higher learning rate. It considers the same parameters of the baseline scenario, but the ecological learning rate is increased to $50 \%$. It is expected that the number of people in the medium environmental awareness group will increase and the low environmental awareness group will decrease in the same proportion.

Scenario 2 (S2): Higher sociability radius. To test the effect of social variables, this scenario keeps most of the parameters of the baseline scenario but increase the maximum sociability radius to 10 .

Scenario 3 (S3): Empirical scenario. We propose a scenario with empirical values, in which an attempt will be made to represent a population composed of $75 \%$ of people in group, $20 \%$ of people with average environmental awareness (medium), and only $5 \%$ of people with high environmental awareness. A maximum sociability radius of 3 is set, the standard deviation of waste generation is increased to $0.3(\mathrm{~kg} /$ hab./ day), the maximum displacement is limited to 4 , and an ecological learning rate of $3 \%$ is established.

The parameters used in each, including the baseline scenario, are summarized in Table II.

\section{A. Scenario analysis}

Each scenario was run 10 times using the Behavior Space tool of the Netlogo software. For all scenarios, the number of people in the NPLe group decreases as the number of people in the NPMe group increases, as we can see in Figure $4(\mathrm{~g})$. At the same time, an increase in the average eco-level of the population is observed, a reduction in the $N W$ as an effect of changing the consumption habits of individuals, while the RWSI is reduced as the RWSC increases. At the end of the simulation, the average eco-level of the population stabilizes to nine, with all humans being part of the third group and adopting the consumption habits defined for it. It should be noted that, the fact that the average eco-level converges to nine might be because all individuals reached the same level, while the 
decision was not to try to persuade their neighbors to increase their eco-level, as it is shown in Figure 4 (j).

In the second scenario, the outputs of the model behave in the same way as in the first scenario up to a certain time. However, because the sociability radius increases the possibility of agents interacting with each other, we find a gradual increase in the NPMe group (see Figure 4 (h)). A change in the average eco-level of the population is also observed in Figure 4 (j). This result is interesting, not only to validate the model, but also to analyze how with only two people trying to generate changes, which represent less than $2 \%$ of the total simulated population, and with a high interaction of agents, it was possible to see a change in a period close to 6 months, even though one of the initial agents was from NPMe group 2, which implied a certain indecision when it came to promoting environmental awareness in the other individuals.

Finally, in the third scenario, as can be seen in Figure 4 (d) and (e), the population becomes part of the NPMe group, while an increase in the amount of recoverable waste separated in a correct way $(R W S I)$ is noted. It is important to mention that the simulated world has a space of $20 \times 20$. To obtain results that are more accurate and perform a deeper analysis, it will be necessary to identify and study a population, establish spatial proportions regarding the physical territory in which the population interacts, and investigate the space-to-person ratio that allows the generation of a more realistic scenario.

\section{CONCLUSIONS AND Future RESEARCH}

Agent-based simulation allows us to describe and analyze different aspects of a system with heterogeneous elements. In the present work, we studied a problem in which environmental awareness, social skills, consumption habits and the amount of waste generated by individuals in a population are related to the planning of extended producer responsibility schemes for packaging. A model was designed to coherently relate the defined concepts, which was described using the ODD + D protocol and validated through the evaluation of three scenarios and a baseline scenario.

It could be concluded that it was possible to relate the identified topics in an agent-based simulation model, which proved to be useful for the study and analysis of this type of problem. Emerging behaviors and influence of each of the defined parameters in the model's results were identified.

In analytical terms, we could say that populations, little by little, increase their average eco-level, if there are people with good social skills and high environmental awareness willing to persuade others. A passive behavior, combined with high resistance to change, can be harmful to a joint state - company - population transition towards a circular economy through extended responsibility schemes. A positive factor not considered in this study is the influence that advertising, social networking, and media campaigns can have on the eco-level of people. These massive persuasive elements could be the subject of further research.

As future work, an information-gathering tool could be created to establish the eco-level of a person and carry out an exploratory study to define the proportions of the number of people per group with real data. In addition, a method to find the percentage of recoverable waste according to their eco-level and their consumption habits could be studied. In the same way, it is important to validate the scales defined with expert opinions to guide the model towards a more realistic approach. Also, this model could be adapted to study customer behaviors in a customer - enterprise system considering new agents. Finally, depending on the population to be studied, it is necessary to define the spatial and agent generation proportions to be used in the simulation.

\section{REFERENCES}

[1] P. R. Ehrlich and J. P. Holdren, "Impact of population growth," Science (80-. )., vol. 171, no. 3977, pp. 1212-1217, 1971.

[2] M. Cropper and C. Griffiths, "The interaction of population growth and environmental quality," Am. Econ. Rev., vol. 84, no. 2, pp. 250254, 1994.

[3] S. Kaza, L. Yao, P. Bhada-Tata, and F. Van Woerden, What a Waste 2.0: A Global Snapshot of Solid Waste Management to 2050. World Bank Publications, 2018.

[4] M. O'Brien, A crisis of waste?: understanding the rubbish society. Routledge, 2012.

[5] M. Lieder and A. Rashid, "Towards circular economy implementation: A comprehensive review in context of manufacturing industry," Journal of Cleaner Production. 2016, DOI: 10.1016/j.jclepro.2015.12.042.

[6] W. R. Stahel, "The circular economy," Nat. News, vol. 531, no. 7595, p. 435, 2016.

[7] Naciones-Unidas, "Objetivos de desarrollo sostenible. 17 objetivos para transformar nuestro mundo. Objetivo 12," 2015.

[8] I. C. Nnorom and O. Osibanjo, "Overview of electronic waste (ewaste) management practices and legislations, and their poor applications in the developing countries," Resour. Conserv. Recycl., vol. 52, no. 6, pp. 843-858, Apr. 2008, DOI: 10.1016/J.RESCONREC.2008.01.004.

[9] A. J. Sinclair, "Assuming responsibility for packaging and packaging waste," Electron. Green J., vol. 1, no. 12, 2000.

[10] D. Bourguignon, "Closing the loop: New circular economy package," Eur. Parliam. Res. Serv., p. 9, 2016.

[11] DNP, "Politica Nacional Para La Gestión Integral De Residuos Solidos," 2016.

[12] Resolución 1407, "Resolución 1407 de 2018. Por la cual se reglamenta la gestión ambiental de los residuos de envases y empaques de papel, cartón, plástico, vidrio, metal y se toman otras determinaciones." Ministerio de Ambiente y Desarrollo Sostenible. 26 Jul, 2018 Núm. 50673, Bogotá D.C., Colombia, 2018.

[13] T. Santa and M. Gonzalez, "Challenges in the implementation of Extended Producer Responsibility policies : The case of Packaging in Chile," pp. 1-53, 2018.

[14] C. Charuvichaipong and E. Sajor, "Promoting waste separation for recycling and local governance in Thailand," Habitat Int., 2006, DOI: 10.1016/j.habitatint.2005.02.002.

[15] J. Pavón, M. Arroyo, and S. Hassan, "Simulación de sistemas sociales con agentes software," Una Perspect. la Intel. Artif. en su 50 aniversario., vol. I, pp. 389 - 400, 2006.

[16] B. Müller et al., "Describing human decisions in agent-based models - ODD + D, an extension of the ODD protocol," Environ. Model. Softw., vol. 48, pp. 37-48, Oct. 2013, DOI: 10.1016/j.envsoft.2013.06.003.

[17] L. Padgham, K. Nagel, D. Singh, and Q. Chen, "Integrating BDI agents into a MATSim simulation," Front. Artif. Intell. Appl., vol. 263, pp. 681-686, 2014, DOI: 10.3233/978-1-61499-419-0-681.

[18] J. Ozik, N. T. Collier, J. T. Murphy, and M. J. North, "The ReLogo agent-based modeling language," Proc. 2013 Winter Simul. Conf. Simul. Mak. Decis. a Complex World, WSC 2013, no. December 2014, pp. 1560-1568, 2013, DOI: 10.1109/WSC.2013.6721539.

[19] B. Argemiro, H. Múnera, A. Adolfo, A. Arrieta, F. Javier, and C. Sierra, "Modelos para el estudio fenomenológico de la combustión sin llama con simulación numérica Numerical models for the 
phenomenological study of flameless combustion," Redalyc, vol. 29, no. 2, pp. 70-76, 2009.

[20] S. Tisue and U. Wilensky, "Netlogo: A simple environment for modeling complexity," Conf. Complex Syst., pp. 1-10, 2004, DOI: 10.1109/ICVD.2004.1261037.

[21] T. Lindhqvist, P. Manomaivibool, and N. Tojo, "La responsabilidad extendida del productor en el contexto latinoamericano," 2008.

[22] S. F. Railsback and V. Grimm, Agent-based and invidual-based modeling, 1st ed. Oxforshire, UK: Princeton University Press, 2012.

[23] S. Abar, G. K. Theodoropoulos, P. Lemarinier, and G. M. P. O'Hare, "Agent Based Modelling and Simulation tools: A review of the stateof-art software," Comput. Sci. Rev., vol. 24, pp. 13-33, 2017, DOI: 10.1016/j.cosrev.2017.03.001.

[24] E. Damiani and G. Sissa, "An agent based model of environmental awareness and limited resource consumption," Proc. 5th Int. Conf. Manag. Emergent Digit. Ecosyst. MEDES 2013, pp. 54-59, 2013, DOI: $10.1145 / 2536146.2536148$.

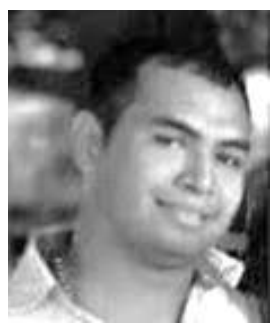

J.D. Galarcio was born in Cereté, Córdoba, Colombia in 1996. He received his degree in Industrial Engineering (B.S.) from the Universidad de Córdoba, Monteria, in 2017. He is a master's degree student (M.S.) at University of Antioquia, Medellin.

From 2016 to 2017, he was a Research Assistant at the Universidad de Córdoba, Monteria. In 2019, he began as a Scholarship Student, performing functions as a Professor of the Industrial Engineering Department at the University of Antioquia in Colombia. He is the author of two research articles and one book, both related to logistics for perishable products, supply chain management and operational research tools. His research interests include optimization, agent-based modeling (ABM), circular economy, metaheuristics, artificial intelligence, and supply chain management.

Ing. Galarcio is an Industrial Engineer at the Universidad de Córdoba (CO). He is a master's in engineering's student at the Universidad de Antioquia in Colombia. His research interests include optimization, agent-based modeling (ABM), circular economy, metaheuristics, artificial intelligence, and supply chain management.

ORCID: https://orcid.org/0000-0002-2192-905X

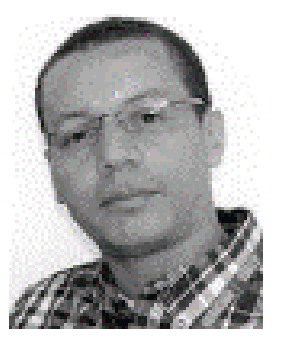

Y.F. Ceballos was born in Guatape, Antioquia, Colombia in 1979. He received the Computer engineering (B.S.), M.S and Ph.D. degrees in computer science from the Universidad Nacional de Colombia, Medellin, in 2004, 2007 and 2015, respectively.

From 2005 to 2013, he was a professor with the computer science Department. Since 2014, he has been an Assistant Professor with the Industrial Engineering Department at Universidad de Antioquia. He is the author of more than 20 articles in the past years. His research interests include numerical methods, game theory, system simulation, behavioral research, algorithms, and stochastic processes.

Dr. Ceballos is an actual member of Society for Industrial and Applied Mathematics (SIAM) and the system dynamics society (SDS).

ORCID: https://orcid.org/0000-0001-5787-8832

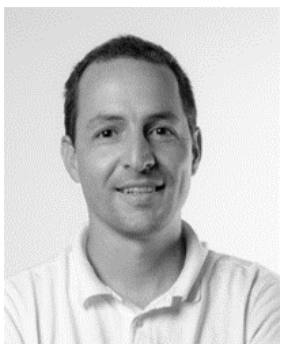

P.A. Maya Duque was born in Medellín, Colombia. He held a B.S. in Industrial engineering (B.S.) from the Universidad Nacional de Colombia a Ms.S in Industrial engineering from the University of los Andes in Colombia and a Ph.D. in Operations research from the University of Antwerp in Belgium.

Dr. Maya is associated Professor at the Industrial Engineering Department of the Antioquia University in Colombia. His research interests are mainly on the field of applied OR techniques, particularly to problems with potential social impact such as circular economy, sustainable transport, health services and humanitarian and non-profit logistics.

ORCID: https://orcid.org/0000-0002-5348-8615

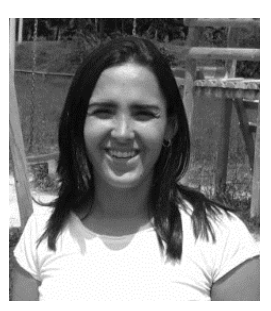

G.L. Ramirez was born in Sucre, Venezuela in 1979. She received the Management Engineering degree from the Universidad Nacional de Colombia, Medellín, in 2003. She holds a Master and a Ph.D. in Accounting and Finance from the Universidad de Zaragoza, España, in 2008 and 2011, respectively.

Dr. Ramirez is a Professor of the Industrial Engineering Department at the Antioquia University in Colombia. Her research interests are related to finance in supply chain management, economic indicators, and circular economy.

ORCID: https://orcid.org/0000-0003-4268-7296 